\title{
Pattern of rheumatic valvular involvement and its contribution towards valvular malfunction in young adults
}

\author{
Arulnithy Kanagasingam 1 , George R Francis², Brinda Komagarajah ${ }^{1}$, Dushjyanthy Ladchumanan 1 , \\ Ahila Sivapramyan', Brindan Packiyarajah', Rasanayagam Megatheepan ${ }^{1}$, Jeyasingam Madura ${ }^{1}$
}

(Index words: rheumatic heart disease, valvular malfunction, mitral valve)

\begin{abstract}
Objective To study the different patterns of valvular malfunction in rheumatic heart disease (RHD) and assess the factors contributing towards it.

Methods This is an observational study among patients with chronic RHD. One hundred patients (female 81 and 19 males) within ages 12 to 40 years (Mean age 27.3) were analyzed. A relevant clinical history including that of an initial episode of acute rheumatic fever (ARF) and recurrent episodes was obtained. 2D echo assessment of the cardiac valves was performed with an estimation of Wilkins score for the mitral valve (MV).
\end{abstract}

Results Among the study population female: male ratio was $4: 1.30 \%$ had recurrent episodes of ARF. Only $60 \%$ had at least some evidence of ARF at any time in their life. The posterior mitral valve appears to be affected more than the anterior leaflet giving an average Wilkins score of 9.7 and 6.7 respectively. The total score had a positive correlation with Mitral stenosis (MS) $(p<0.05)$. MV involvement was noted in $97 \%$. $44 \%$ had significant mitral valve prolapse (MVP) but no statistical correlation was noted with mitral regurgitation $(M R)(p>0.05)$. A regurgitant grade of 2 or more was found in $41 \%$. High sensitive $C$ reactive protein of more than $1 \mathrm{mg} / \mathrm{dl}$ was noted in $55 \%$ of patients.

Conclusion Chronic rheumatic MV disease can exist as MS, MR, MVP or simply an elevated valve score. Apart from recurrent streptococcal infections and chronic sub clinical inflammation, a number of different components of valve damage contribute towards the end result.

Ceylon Medical Journal 2019; 64: 91-97

DOI: http://doi.org/10.4038/cmj.v64i3.8951

\section{Introduction}

RHD is still a disease, causing significant mortality and morbidity in the developing world. It has been estimated that there are 15.6 million people affected by RHD. And every year 23300 deaths occur attributed to RHD [1-3]. Rheumatic fever is a multisystemic autoimmune disease resulting from infection with group A streptococcus. Morbidity and mortality vary from region to region worldwide, and is high in Asia [2] including Sri Lanka. Chronic RHD cause significant valvular damage needing valve replacement and most often valve intervention. Persistent inflammatory damage and hemodynamic injury to the valve leaflets are the main contributors towards the gradual progression of the disease [4]. Key anatomic abnormalities are leaflet thickening, nodularity, calcification, and commissural fusion, although all of these eventually result in narrowing of the valve orifice, their degree of contribution may not be the same. MS progresses much more rapidly in the background of severe or recurrent infections [5]. There are several studies describing the mechanisms and manifestations of valve damage in ARF but only limited studies look into chronic RHD. There are no studies looked at the factors that favor the type of valvular damage, and only a few studies assess the pattern of valvular involvement in chronic RHD. We designed this study to identify the different types of valvular malfunctions in chronic RHD. We also looked into non-valvular and valvular factors which could influence the degree of valvular malfunction.

Valve damage was objectively assessed by means of 2D Echocardiography. Factors already known to cause or take part in progression of valve damage according to available literature were also assessed.

${ }^{1}$ Cardiology Unit, Teaching Hospital Batticaloa, ${ }^{2}$ Faculty of Health-Care Sciences, Eastern University, Sri Lanka.

Correspondence: KA, e-mail: <karulnithy@yahoo.co.uk>. Received 16 April 2019 and revised version 11 July 2019 accepted 24 September 2019.

This is an open-access article distributed under the terms of the Creative Commons Attribution License, which permits unrestricted use, distribution, and reproduction in any medium, provided the original author and source are credited. 


\section{Methodology}

\section{Study design and setting}

This is a cross-sectional study, performed over the period of March 2016 to November 2017. Participants were diagnosed RHD patients aged above 12 years and not more than 40 years of age, following the Rheumatic Clinic at the Cardiology Unit, Teaching Hospital, Batticaloa, Sri Lanka. The sample size was one hundred. The size was determined according to similar studies done in the past. Patients with other co-morbid diseases, those with acute rheumatic fever and patients who had undergone valve replacement were excluded.

\section{Data Collection}

Data collection is done by a Consultant Cardiologist, Medical Officers, and Research Assistant. Information regarding demographic details, clinical history and follow up data was collected via an interviewer-administered questionnaire, clinic notes and laboratory investigations. A 2D echocardiogram was done by a Consultant Cardiologist and recorded. Wilkins score was calculated based on an assessment of leaflet mobility, valve thickening, calcification, and sub-valvular involvement. Scores relating to the anterior mitral valve leaflet (AMVL) and the posterior mitral leaflet (PMVL) were calculated separately. Each component was graded from 0 to 4 . A normal valve score will be " 0 " in contrast a severely damaged valve will carry a maximum score of "16". The average of both leaflet scores was taken as the final MV score. MV area was determined by circumferential tracing of the MV leaflet edges at maximal opening during diastole, and doppler mitral valve area using the pressure half time. Furthermore, MVP was determined by the systolic displacement of the coaptation point posterior to the annular plane in the long axis. Pressure gradients across the MV, degree of MR and commissural fusion were also assessed. Aortic and Tricuspid valves were also assessed for stenosis and regurgitation using hemodynamic parameters. MR and aortic regurgitation (AR) were diagnosed by using standard protocols. The involvement of the tricuspid valve (TV) in RHD was determined by the presence of thickening and sclerosis. Tricuspid pressure gradient (TRPG) of more than $25 \mathrm{mmHg}$ at rest was taken to indicate significant pulmonary hypertension.

\section{Ethical Consideration}

Ethical clearance was obtained for the research from the Ethical Review Committee, Faculty of Healthcare Sciences, Eastern University, Sri Lanka. (ID: EUSL/FHCS/ ERC/2016/04). Participants were informed of the study and written consent was obtained. Consent was obtained from the parent or guardian for patients below 18 years of age. Verbal consent was obtained for blood sampling. All consent forms have been produced in their mother tongue.

\section{Data Analysis}

Collected data was entered in a computer and analyzed by SPSS (Statistical Package of Social Sciences $-20^{\text {th }}$ version). The normality of data distribution was tested using the Kolmogorov-Smirnov Test. To determine the correlation between trace mitral valve area (MVAT), area by MV pressure half time, and gradient across MV, values were transformed to a normal distribution by using the logarithm of their value. Relationship between variables were assessed using parametric tests and nonparametric tests according to the distribution of data. Statistical significance was taken at $\mathrm{p}<0.05$.

\section{Results}

The study included 100 patients identified as RHD in whom $2 \mathrm{D}$ echocardiogram was done to determine the characteristics of the disease. The mean age was $27.3 \pm$ 7.3 years, 81 were female. Majority $(92 \%)$ were from rural or suburban areas.

Among the patients with a history of an acute episode of RHD, the prevalence of joint involvement $(73.3 \%)$ and fever $(71.7 \%)$ were the common clinical findings. Only one patient had documented subcutaneous nodules with fever and sore throat at presentation. Two patients had abnormal body movements suggestive of chorea, one patient didn't have any described symptoms or signs. Forty percent of the population had no history of ARF. Around 30\% of the population had evidence of recurrent episodes of ARF. Nine of them had recurrences while on penicillin prophylaxis. 2D echocardiographic findings are summarized in Table 1.

\section{Correlation between different parameters}

Variables MVAT, MV pressure half time (MV P1/2t) and MV pressure gradient were transformed into normal distribution by using the logarithm of their value. Correlation between MVAT and mean pressure gradient across the mitral valve was found to have statistically significant correlation $(\mathrm{r}=-0.755, \mathrm{P}<0.001$ Fig 3 -B). MV $\mathrm{P} 1 / 2 \mathrm{t}$ was positively correlated with MVAT $(\mathrm{r}=0.826$, p $<0.001$, Fig. 3-A). Severity of MS detected by different means such as Trace MV area, Mean PG across MV, Pressure half time showed considerable variation in detection of severity (Fig 2). Correlation of mitral valve leaflet mobility $[\mathrm{r}=0.68, \mathrm{~F}(1,98)=11.92, \mathrm{p}<0.001]$, leaflet thickening $[\mathrm{r}=0.32, \mathrm{~F}(1,98)=11.92$, p 0.005), calcification $[(r=0.52, F(1,98)=36.65 \mathrm{p}<0.001]$, and subvalvular thickening $[\mathrm{r}=0.66, \mathrm{~F}(1,98)=77.51, \mathrm{p}<0.001]$, were correlated with MVAT. Among all, immobility and sub-valvular thickening possessed the strongest correlation with MS severity. On linear regression the average mitral valve score had negative correlation with MVA Trace [ $r=-0.662, \mathrm{p}<0.001]$, and severity of MR $[r=-0.076, P>0.05]$. A Kendall's tau-b correlation was run to determine the relationship between the average mitral 
valve score and the presence of mitral valve prolapse. The relationship was weak and not statistically significant $\left[\tau_{b}=-0.077, \mathrm{P}=0.364\right]$. There was a significant positive correlation noted between average valve score and severity of MS but not with MR.

Table 1. Complete 2D Echocardiogram findings with frequencies/ percentage

\begin{tabular}{|c|c|}
\hline Findings & $n / \%$ \\
\hline \multicolumn{2}{|l|}{ Severity of mitral stenosis } \\
\hline None & 33 \\
\hline Mild & 41 \\
\hline Moderate & 17 \\
\hline Severe & 9 \\
\hline \multicolumn{2}{|l|}{ Severity of mitral regurgitation } \\
\hline None & 6 \\
\hline Grade I & 53 \\
\hline Grade II & 32 \\
\hline Grade III & 8 \\
\hline Grade IV & 1 \\
\hline \multicolumn{2}{|l|}{ Severity of aortic stenosis } \\
\hline None & 93 \\
\hline Mild & 6 \\
\hline Moderate & 1 \\
\hline Severe & 0 \\
\hline \multicolumn{2}{|l|}{ Severity of aortic regurgitation } \\
\hline None & 54 \\
\hline Grade I & 31 \\
\hline Grade II & 13 \\
\hline Grade III & 2 \\
\hline \multicolumn{2}{|l|}{ Presence of mitral valve prolapse } \\
\hline Yes & 44 \\
\hline No & 56 \\
\hline \multicolumn{2}{|l|}{ Lateral commissural fusion } \\
\hline Not fused & 43 \\
\hline Partially fused & 31 \\
\hline Fused & 26 \\
\hline \multicolumn{2}{|l|}{ Medial commissural fusion } \\
\hline Not fused & 57 \\
\hline Partially fused & 25 \\
\hline Fused & 18 \\
\hline \multicolumn{2}{|l|}{ Tricuspid valve involvement } \\
\hline Yes & 10 \\
\hline No & 90 \\
\hline \multicolumn{2}{|l|}{ Pulmonary hypertension } \\
\hline Significant TRPG & 33 \\
\hline Non-significant TRPG & 67 \\
\hline Presence of myxomatous mitral valve (n/\%) & 12 \\
\hline \multicolumn{2}{|c|}{ Involvement of heart valves in participants } \\
\hline MV involvement (n/\%) & 97 \\
\hline AV involvement $(\mathrm{n} / \%)$ & 46 \\
\hline
\end{tabular}

The association between the presence of MVP and MR was not statistically significant $(\mathrm{P}>0.05)$. No relationship has been observed for the presence of MVP with PMVL score or PMVL mobility among participants $(\mathrm{P}>0.05)$.

Among the sample 94 of them had some degree of MR but only 41 of them had significant MR (Grade $\geq$ II), 68 participants had MS, 20 participants had significant MR without MS. Patients undergoing PTMC had a statistically positive correlation with valve score $\left(\mathrm{r}_{(\mathrm{s})}=0.489, \mathrm{P}<0.005\right)$.

\section{Discussion}

Our study showed MS, MR, MVPAS and AR are the usual forms of valvular malfunction in RHD. There are already known factors which could influence the progression of valve damage also noted in our study. Factors like female predominance, rural and sub urban population, where internal overcrowding is common were prominent in our population too. These were known risk factors in ARF and RHD [6,7]. Recurrent infections were documented in $30 \%$ of the population similar to previous studies $[11,12]$. Among these patients, the majority gives the history of defaulting penicillin prophylaxis.

A significant number of patients (40\%) had no clinical event indicating a history of acute rheumatic fever in the past. This shows that these patients probably had a clinically silent acute episode. Mild or even asymptomatic ARF has been reported in the literature but not to this extent [8,9]. Clinically silent Chronic RHD is well documented [10]. Because secondary prevention is the most important factor to prevent the progression of RHD, present criteria to identify ARF needs significant amendment to detect these clinically silent patients. Our findings are also not consistant with the opinion that less severe ARF does not cause cardiac involvement [11]. In fact, there could be a lot more people within the community with Chronic RHD especially when they have clinically silent disease. Effective measures need to be proposed such as population-based 2D echocardiographic screening to identify these people for secondary prophylaxis [10].

\section{D Echocardiographic pattern of valvular involvement}

Forty-six patients (46\%) had AV involvement. Among these AS was detected only in 7 patients. There were no cases of severe AS. Fifteen patients had significant AR of more than Gr II (Table 1). These findings are similar to previous studies $[10,13]$.

As the mitral valve is the commonest target for RHD, the focus of this study was an in depth assessment of the mitral valve. MS with commissural fusion and MR were the clinically important end results of MV involvement. Since MVP is also a known feature of chronic RHD [14], MVP and its impact on MR was also assessed. Wilkins score was used to assess the severity of MV involvement [15], even though the Wilkins score was originally 
formulated to assess the suitability of percutaneous mitral commissurotomy (PTMC), we considered it reasonable to use this score as it covers the major anatomical features of valvular involvement.

We found that the PMVL encountered significant damage when compared to the AMVL (Average score of AMVL: PMVL=6.7:9.7). (Fig 1). This could be due to the different morphology of the PMVL compared to AMVL, at the same time predilection of PMVL compared to the AMVL in RHD cannot be excluded. MS is a welldocumented sequel of RHD. The majority had only mild or moderate MS (58\%) in the study group. This could be due to the fact that, patients with severe MS had undergone PTMC, Mitral valve replacement or died due to complications of severe valve involvement.

While assessing the severity of MS usually the trace $\mathrm{MV}$ area is considered more accurate than MV P1/2t or PG. Among both of these measurements, we found that MV $\mathrm{P} 1 / 2 \mathrm{t}(\mathrm{r}=0.826)$ correlated better with traced values than $P G(r=0.755)$. In situations where tracing the valve became difficult such as poor windows, severely fibrosed or calcified valves where edges are too difficult to define, MV P1/2t is more desirable than PG (Fig 3).

More than half of the population had partially or completely fused commissures and this was one of the significant factors which promote clinically significant valvular damage in the form of MS. Immobility and subvalvular thickening had a strong correlation with severity of MS. Both these parameters should be given more weight on predicting future MS. There was also a significant positive correlation noted between the average valve score and severity of MS but not with MR. There could be several other anatomical factors involved in the manifestation of MR not merely a severity of the valve damage. Isolated MR in patients with a history of RHD is well documented in the literature [16, 17]. Significant MR (Grade $\geq$ II) alone without MS was detected in 20 patients. Only one had very severe MR (Grade IV). Severe regurgitation (Grade III, IV) is much low compared to severe MS. MR is a prominent finding in acute RF than MS. Mainly due to annular dilatation and MVP [22].

We found a significant population with $\operatorname{MVP}(44 \%)$, (Table 1) of AMVL. This could be due to immobility of PMVL, shortening of the PMVL chordae, and possibly due to elongation of AMVL chordae as in ARF [22]. They might progress to MR later years. Follow-up studies are needed to assess the fate of MVP. But in our study no statistically significant relationship of MVP and the presence of MR was noted ( $\mathrm{P}>0.05)$ unlike in ARF [22]. This could be due to the fact that with time AMVL prolapse is reduced due to shortening of chordae so the MR simply could be due to mal-coaptation rather than MVP in chronic RHD patients. Progressive PMVL damage could be a causative factor for MVP, and MVP does not appear to be responsible for or act as a precursor of progressive MV damage.

\section{Inflammation and valve damage}

There is documented evidence to show that there is a progressive immune response in the chronic phase of RHD [18]. There is also evidence to say that oxidative stress, hsCRP could be involved in the pathogenesis of rheumatic heart valve disease [18, 19, 21]. Persistent elevations of CRP has been noted in chronic rheumatic heart valve disease [20].

Evidence of chronic inflammation was checked by measuring C-reactive protein (CRP) and hsCRP. High CRP or hsCRP (>1mg/L) was noted in $56.8 \%$ of subjects out of 88 patients in which hsCRP was measured. But no statistically significant correlation was found between the CRP and severity of $\mathrm{MS}\left(\mathrm{r}_{(\mathrm{s})}=0.018, \mathrm{P}>0.05\right), \mathrm{MR}\left(\mathrm{r}_{(\mathrm{s})}=\right.$ $0.172, \mathrm{P}>0.05)$, presence of $\mathrm{MVP}\left(\mathrm{r}_{(\mathrm{s})}=-012, \mathrm{P}>0.05\right)$, presence of commissural fusion (Lateral commissure; $r_{(\mathrm{s})}$ $=0.087, \mathrm{P}>0.05$, medial commissure $\mathrm{r}_{(\mathrm{s})}=0.12, \mathrm{P}>0.05$ ) or the total valve score. Effects of persistently elevated CRP and valve damage needs to be observed in long term follow-up.

Interestingly the level of hsCRP was significantly lower in patients who had undergone PTMC when compared to the rest of the population (Table 3). This favors the fact that turbulent flow due to a damaged valve could be the major factor, which leads to progressive fibrosis, thickening, and calcification. Hemodynamic turbulence could be the cause for the persistent subclinical inflammation, with PTMC gradient across MV is reduced significantly. hsCRP also appears significantly higher in patients with multi-valvular involvement indicating an excess of subclinical inflammation [20].

Table 2. Presence of MVP and MR

\begin{tabular}{|c|c|c|c|c|c|}
\hline & \multicolumn{5}{|l|}{$M R$} \\
\hline & $\begin{array}{l}N o \\
M R\end{array}$ & $\begin{array}{c}\text { Grade } \\
I\end{array}$ & $\begin{array}{c}\text { Grade } \\
\text { II }\end{array}$ & $\begin{array}{c}\text { Grade } \\
\text { III }\end{array}$ & $\begin{array}{c}\text { Grade } \\
I V\end{array}$ \\
\hline MVP present & 2 & 23 & 15 & 3 & 1 \\
\hline No MVP & 4 & 30 & 17 & 5 & 0 \\
\hline
\end{tabular}

Table 3. PTMC vs CRP (PTMC - Percutaneous trans mitral commissurotomy; CRP - C reactive protein)

\begin{tabular}{llcccc}
\hline \multirow{5}{*}{} & \multicolumn{4}{c}{ CRP/ hsCRP } \\
\cline { 2 - 6 } & \multicolumn{1}{c}{$<1 \mathrm{mg} / \mathrm{L}$} & $1-3 \mathrm{mg} / \mathrm{L}$ & $3-10 \mathrm{mg} / \mathrm{L}$ & $>10 \mathrm{mg} / \mathrm{L}$ \\
\hline PTMC & Done & 8 & 8 & 4 & 0 \\
& Not done & 30 & 16 & 17 & 6 \\
\hline
\end{tabular}




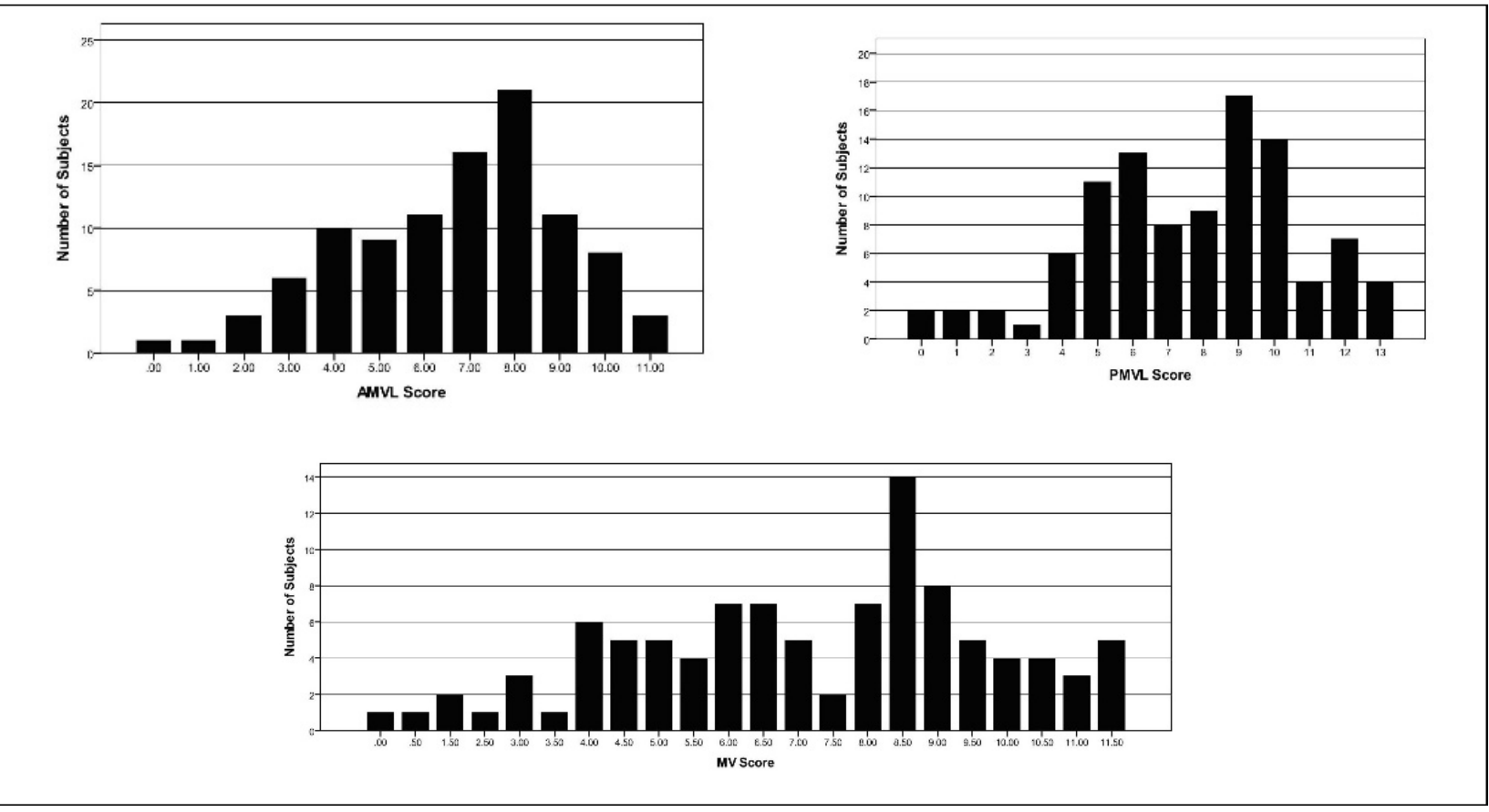

Figure 1. Frequencies of mitral valve score. Left: AMVL (Anterior mitral valve) score, Right: PMVL (Posterior mitral valve) score, Bottom: Average MV (mitral valve) score.

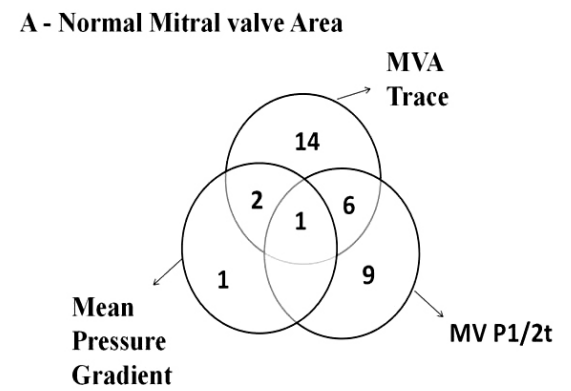

C - Moderate Mitral Stenosis

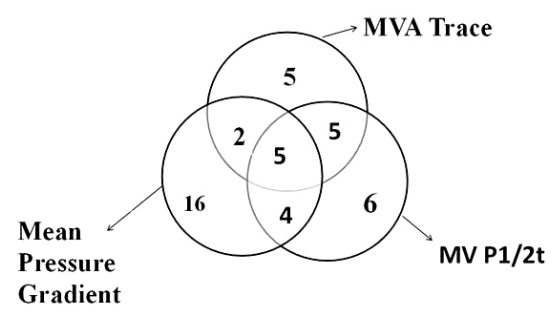

B - Mild Mitral Stenosis

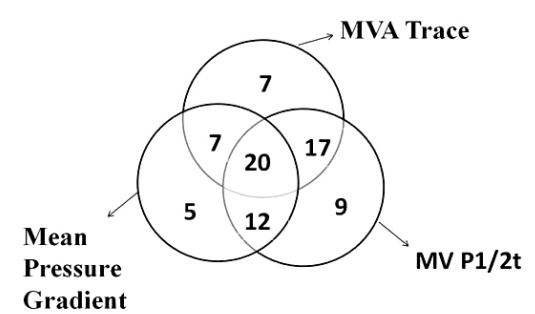

D - Severe Mitral Stenosis

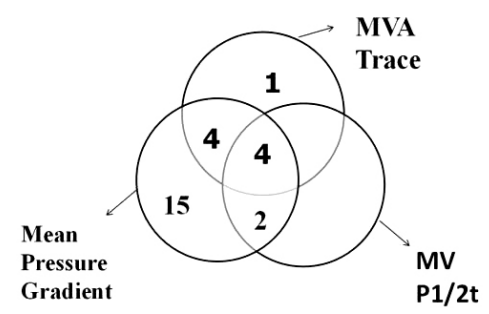

Figure 2. The severity of MS (Mitral stenosis) detected via different techniques (Trace MV area, Mean Pressure Gradient across MV, Pressure half time (MV P1/2t)). Frequency of participants with A- Normal MVA (Mitral valve area), B- Mild MS, C- Moderate MS, D- Severe MS. 
A

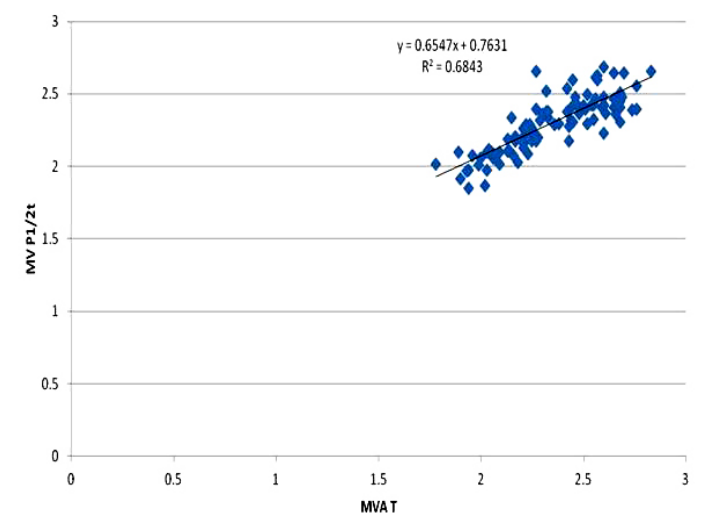

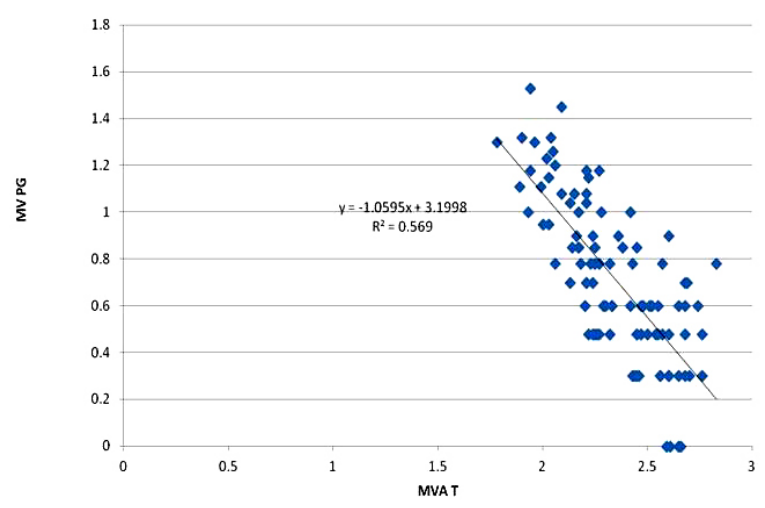

Figure 3. Correlation between MVAT (Trace mitral valve area) and area by MV P1/2t (Pressure half time) (left), Correlation between MVAT (Trace mitral valve area) and area by MV PG (Mean pressure gradient across) (right).

The statistically positive correlation of PTMC with valve score $\left(\mathrm{r}_{(\mathrm{s})}=0.489, \mathrm{P}<0.005\right)$ could be the result of mechanical damage which heals by fibrosis and scarring without persistent inflammation. Usually, PTMC is attempted in patients with a Wilkins score of less than or equal to eight. Inflammatory markers just after PTMC might give a clue of post-PTMC healing and scaring.

In addition to factors like recurrent streptococcal infection, valvular changes identified by $2 \mathrm{D}$ echocardiography and sub clinical inflammation have some influence on valvular malfunction. A detail prospective study would shed more light on these issues.

\section{Conclusion}

The sub-clinical nature of the acute and chronic stage is the major challenge in identifying patients with RHD. One needs to consider MS, MR, MV score and MVP separately in assessing the severity of rheumatic MV disease. Relatively high score of PMVL, presence of MR, AR and MVP might help to identify the RHD patients in routine 2D Echocardiogram in the age group of 12 to 40 years. hsCRP would help as a blood investigation in this aspect. Commissural fusion, sub-valvular involvement, and immobility of leaflets are other factors which could predict future significant MS. Factors producing a MR in Chronic RHD needs further investigation.

\section{Acknowledgments}

We like to express our sincere thanks to Dr Krishanthi, Dr Goutham and the Echo lab staff.

\section{Conflict of interests}

The are no conflicts of interest. No financial support was obtained for this study.

\section{References}

1. Carapetis, Jonathan R., Malcolm McDonald, and Nigel J. Wilson. Acute Rheumatic Fever. Lancet 2005; 366: 155-68.

2. WHO Study Group on Rheumatic Fever and Rheumatic Heart Disease (2001: Geneva, Switzerland) \& World Health Organization. (2004). Rheumatic fever and rheumatic heart disease: report of a WHO expert consultation, Geneva, 20 October - 1 November 2001: 1-122. World Health Organization.

3. Essop, Mohammed Rafique and Vuyisile T. Nkomo. Rheumatic and Nonrheumatic Valvular Heart Disease: Epidemiology, Management, and Prevention in Africa. Circulation 2005; 112(23): 3584-91.

4. Nulu S, Bukhman G, Kwan GF. 'Rheumatic Heart Disease: The Unfinished Global Agenda' . Cardiol Clin 2017: 165-80.

5. Selzer A, Cohn KE. n.d. Natural History of Mitral Stenosis: A Review. Circulation 1972; 45: 878-90.

6. Carapetis JR, Wolff DR, Currie BJ. Acute Rheumatic Fever and Rheumatic Heart Disease in the Top End of Australia's Northern Territory. Med J Aust. 1996: 164(3): 146-49.

7. Jaine, Richard, Michael Baker, et al. Acute Rheumatic Fever Associated with Household Crowding in a Developed Country. Pediatr Infect Dis J 2011: 30(4): 315-19.

8. Narula J, Virmani R, Reddy K, et al. Rheumatic Fever. Washington, DC: American Registry of Pathology;1999.

9. Bishop W, Currie B, Carapetis J, et al. Subtle presentation of acute rheumatic fever in remote northern Australia. Aust N Z J Med. 1996; 26: 241-242.

10. Marijon, Eloi et al. Prevalence of Rheumatic Heart Disease Detected by Echocardiographic Screening. $N$ Engl J Med 2007; 357(5): 470-76.

11. Lawrence, Joanna G, Jonathan R, Carapetis, et al. Acute Rheumatic Fever and Rheumatic Heart Disease: Incidence and Progression in the Northern Territory of Australia, 1997 to 2010. Circulation 2013; 128(5): 492-501. 
12. Antonini-Canterin, Francesco et al. Hydroxymethylglutaryl Coenzyme-A Reductase Inhibitors Delay the Progression of Rheumatic Aortic Valve Stenosis. A Long-Term Echocardiographic Study. J. Am. Coll. Cardiol 2009; 53(20): 1874-79.

13. Sani, Mahmoud U, Kamilu M, Karaye, et al. Prevalence and Pattern of Rheumatic Heart Disease in the Nigerian Savannah: An Echocardiographic Study. Cardiovasc J Afr 2007; 18(5): 295-99.

14. Lembo NJ, et al. Mitral Valve Prolapse in Patients with Prior Rheumatic Fever. Circulation 1988; 77(4): 830-36.

15. Wilkins, Gerard T, Arthur E, Weyman, et al. Percutaneous Balloon Dilatation of the Mitral Valve: An Analysis of Echocardiographic Variables Related to Outcome and the Mechanism of Dilatation. Heart 1988; 60(4): 299-308.

16. Jhaveri S, Czoniczer G, Reider RB, et al. Relatively benign "ipure" mitral regurgitation of rheumatic origin: a study of seventy-four adult patients. Circulation 1960; 22: 39.

17. Selzer A, Katayama F. Mitral regurgitation: clinical patterns, pathophysiology and natural history. Medicine 1972; 51: 337.

18. Chopra, Prem and Hanni Gulwani. Pathology and
Pathogenesis of Rheumatic Heart Disease. Indian J Pathol Microbiol. 2007; 50(4): 685.

19. Davutoglu, Vedat, Ahmet Celik, et al. Contribution of Selected Serum Inflammatory Mediators to the Progression of Chronic Rheumatic Valve Disease, Subsequent Valve Calcification and NYHA Functional Class. J Heart Valve Dis 2005; 14(2): 251-56.

20. Gölbasi, Zehra, et al. Increased Levels of High Sensitive CReactive Protein in Patients with Chronic Rheumatic Valve Disease: Evidence of Ongoing Inflammation. Eur J Heart Fail. 2002; 4(5): 593-95.

21. Chiu-Braga YYSY, Hayashi, M Schafranski, et al. Further Evidence of Inflammation in Chronic Rheumatic Valve Disease (CRVD): High Levels of Advanced Oxidation Protein Products (AOPP) and High Sensitive C-Reactive Protein (Hs-CRP). Int J Cardiol. 2006; 109(2): 275-76.

22. Reményi et al. World Heart Federation criteria for echocardiographic diagnosis of rheumatic heart disease-an evidence-based guideline. Nat Rev Cardiol 2012; 9(5): 297-309. 